\title{
Identification of Sickle Cells in Erythrocytes Images Using Shape Descriptors
}

\author{
L.Yamini ${ }^{\mathrm{a}, 1}$, R.Priyadharsini ${ }^{\mathrm{b}}$ and A. Beulah ${ }^{\mathrm{c}}$ \\ ${ }^{a, 1} U G$ Student, Dept of CSE, SSN College of Engineering, India \\ ${ }^{b}$ Associate Professor, Dept of CSE, SSN College of Engineering, India \\ ${ }^{c}$ Assistant Professor, Dept of CSE, SSN College of Engineering, India
}

\begin{abstract}
Sickle cell anemia is a blood disorder which is widespread across world with an estimate that about $30 \%$ of total population will be affected by the end of 2050. Especially in India, the frequency of sickle cell trait can reach up to $35 \%$, thereby increasing attention over the topic for research. It is necessary to detect the disorder as much as it is for finding a cure to it. Therefore, a system that detect the sickle cells from the blood smear images or erythrocytes images is required. The affected cells change their shape from circular to sickle shape, due to the presence of typical hemoglobin called hemoglobin S. Nowadays, the elongated affected cells are identified using image processing techniques. The shape descriptors which are the vital features used to identify any shapes in an image. The contrast and brightness of blood smear images may not be consistent as the acquisition of these images depends on various factors like luminance and chrominance. To detect the sickle cells properly in the blood smear images, the histogram equalization technique is applied to improve the contrast of the image. The image is converted to binary in order to find the boundary of the cells in the image. In the binary image, each normal cell will have holes, Since the normal red blood cell have the shape of a doughnut that has been pressed in the middle slightly. The boundary of all the cells in the image is traced and the holes inside each cells are filled. Each cell is considered as a connected component for which the eccentricity is calculated. The eccentricity is the shape descriptor which is the ratio of the major axis and minor axis of the connected components. If the eccentricity is greater than threshold, the cells are identified as sickle cells.
\end{abstract}

Keywords: Anemia, Connected Component, Eccentricity, Sickle.

\section{Introduction}

Sickle Cell Disease (SCD) is the most common inherited blood disease that results in many complications due to lack of oxygen delivery by sickle cells. SCD can lead to complications such as Anemia, infections, delayed growth, and episodes of pain, called pain crises. To prevent these complications, early diagnosis and treatment of

${ }^{1}$ R.Priyadharsini, Associate Professor, Dept of CSE, SSN College of Engineering, Chennai, India.

E-mail: priyadharsinir@ssn.edu.in 
Sickle cell disease is vital. Sickle cells are those red blood cells that go out of shape and become sickle-shaped (like a crescent moon) instead of their normal disc shape. If both parents have sickle cell trait in their genes, their children could get a double dose of the sickle cell gene, which would give them SCD. There are many ways for detecting sickle cells, which include manual detection and Computer Aided Diagnosis (CAD). The manual detection methods are highly prone to errors and require tedious efforts. The commercially available hematology analyzers which work on the principle of SCD is required. In image processing, there are many object detection techniques available, that can be used for many applications. The techniques used for detection are dependent on the nature of the images being applied to. The object detection in an image can be done using edge detection techniques, shape detection using Hough transform, shape descriptors and texture descriptors. This paper provides a more reliable and an accurate method for detecting sickle cells using shape descriptors.

\section{Data Acquisition}

The images of blood cells are captured using the cell analyser imaging system. The components of the system includes (i) Stabilized light source to illuminate the blood sample, (ii) Motorized stage to move the object into the microscope's field of view, (iii)Microscope optics to magnify the image, (iv) Camera to acquire the images, (v) Digitization process for transforming the video signal into digital format. The blood smear images are examined under oil immersion and are captured in the JPEG format at the maximum resolution.

\section{Literature Survey}

Sickle cell disease being a common and prominent problem since early 90 s has brought attention to various research work in detecting the disease either manually or automatically using image processing techniques to clearly visualise. Even though World Health Organization (WHO) handles the prevention and management of SCD, only minor action is followed. This bought attention to various research works, either manual or automatic detection of SCD. New-borns and occasionally their blood relations will undergo High Performance Liquid Chromatography (HPLC), to separate the blood compounds. Applying Imaging Flow Cytometer in the anticoagulated peripheral venous blood, detects the sickle cells [1]. The authors developed software to classify the normal and sickle cells.After, a series of manual screening of individual blood samples then enter the automatic analysis and classification of different blood cells. The analysis of the blood cells results based on the five different features such as size, roundness, circularity, eccentricity and central gray level distribution. The area, perimeter, diameter, Shape Geometric Factor (SGF), Area proportion (AP), and Deviation value (DV) distinguishes these classes [2]. The DV is the ratio between the SGF and area. If the DV is greater than 0.2 , the cell is classified as sickle cell.

Weiner filter along with sobel edge detection technique extracts the boundary of the blood cell [3]. Ellipse adjustment technique analyses the shape of the erythrocytes [4]. Another paper uses Fractal Dimension using box counting technique in recognizing the sickle cells shape [5]. Image enhancement method hue saturation, median filtering and contrast stretching segments the red blood cells in the given sample[6].Several 
machine learning techniques are adopted in detecting sickle cells. Adapted grey world normalization method performs a better colour normalization [7]. The colour normalization reduces the illumination effect in the images. Morphological operators are used in segmenting the different blood cells. Each segmented blood cell is given as input to 3 and 4 layer back propagation neural networks. This system shows a very low error rate. They have applied morphological operations for enhancing the images. Knearest neighbour classifier classifies the different blood cells based on the features such as aspect ratio, metric value, variance, and radial signature. Circular Hough Transform (CHT) distinguishes the normal and abnormal cells. The neural network and regression tree diagnose the number of sickle cells in the image [8]. Further some study uses Support Vector Machine (SVM) to classify the cells. It uses optimization segmentation, edge smoothing with mean filter to get the features of the blood cells. The classification is done using SVM [9]. Another paper suggests red blood cell classification method for the SCD based on pre-extraction of the cell region and deep Convolution Neural Networks (dCNNs) [10-14].

\section{Proposed System}

There are many object detection technique available in image processing area which are used for identifying the location, shape and type of objects in an image. The object detection methods are more image specific that is they are dependent on the input being used. The edge detection techniques such as Sobel, Robert, Prewitt, Cannyetc are traditional object detection methods. These methods give the outline of the objects however the output may have false edges. Hough transform is a better option for detection of lines, circles, ellipses and any arbitrary objects in an image. It identifies the features in the Hough space which is very useful even when the edge points are not contiguous, and also can handle noisy data. The transforms may also give misleading results when objects happen to be aligned by chance. These may take too much storage space and time as they detect in Hough space. Texture descriptors are also used for boundary representation. There are statistical, structural and spectral approaches exist for describing the texture of the images. However these methods when applied to blood smear images does not detect the sickle cells accurately. Hence an automatic system that detects the sickle cells from the blood smear image is proposed. The shape descriptors are the essential features that can identify the shape of the sickle cells. In this work, shape descriptors are used to identify the affected cells. Figure 1.shows the process of sickle cell detection.

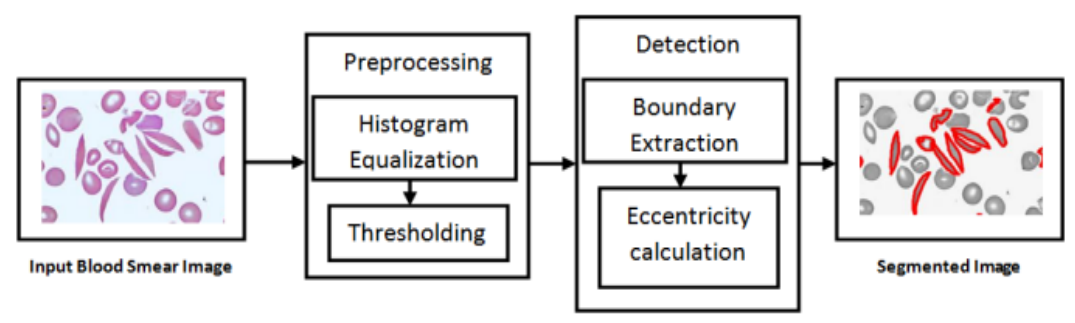

Figure 1.Sickle cell detection process 


\subsection{Contrast Enhancement}

Factors such as illuminance and chrominance affect the contrast and brightness of blood smear images. In order to detect the shape of the sickle cells, preprocessing of the images is required. Preprocessing techniques include the contrast enhancement methods used for improving the intensity values of the images. Histogram equalization is the enhancement technique used in the pre-processing stage for improving the global contrast.

\subsection{Shape descriptors}

In image processing applications such as object matching, identification and classification, the shape based techniques are widely used. There are area based, boundary based and component based shape descriptors that can be used for object detection and identification. The area based shape descriptors are used when the objects in the image seem to be large. If the objects are very small and not distinguishable from the background, boundary based approaches are generally used. In sickle cell detection, the small sickles are identified by tracing the boundary of the objects.

\subsection{Eccentricity}

The contrast of the blood smear images are improved using histogram equalization. To find the boundary of the cells in the image, the contrast enhanced image is converted to binary image. As the normal red blood cells have the shape of a doughnut, holes will be present in all the normal cells. In order to identify the sickle cells from the normal ones the holes are filled. The cells in the image are considered as the connected component and the eccentricity is calculated for each cell. The ratio of the major axis and minor axis of the connected components gives the eccentricity. A threshold is set to identify the sickle cells from the normal cells.

\section{Experimental Result}

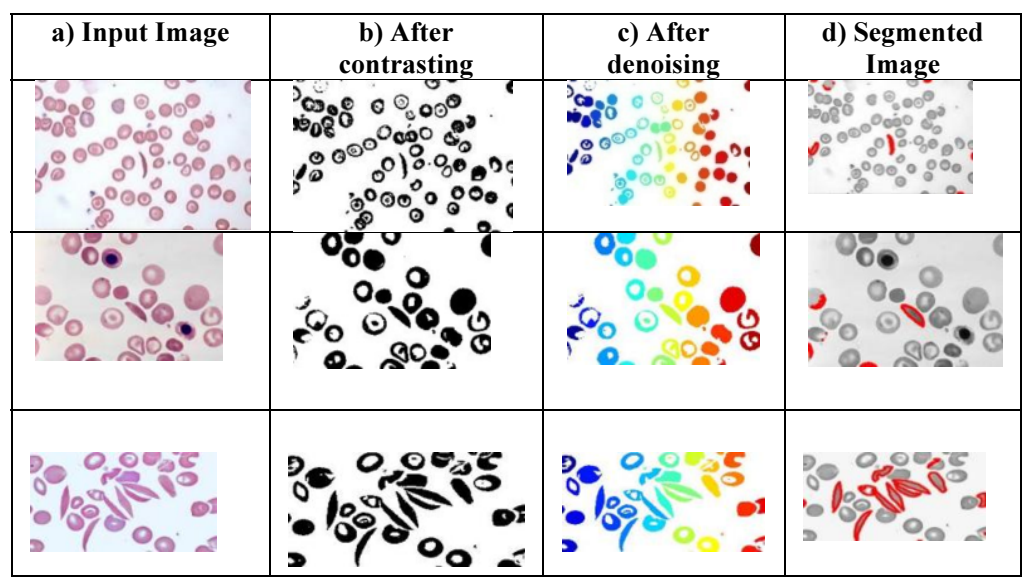

Figure 2.Experimental results of contrasting, denoising and segmentation 


\section{Conclusion}

In this paper, identification of sickle cells from blood smear images have been proposed using shape descriptors. The images generally lack in illuminance and chrominance, so all the images are pre-processed before detecting the sickle cells. The histogram equalization is applied to enhance the images and they are converted to binary images. The boundary of the cells are traced by shape descriptors and the eccentricity is calculated. The holes in the normal red blood cells are filled and a threshold is set to detect the sickle cells. The work has been implemented using MATLAB 9.8 version. The experimental results prove that the method has distinctly identified the sickle cells in the blood smear images.

\section{References}

[1] Van Beers, Eduard J., Leigh Samsel, Laurel Mendelsohn, RehanSaiyed, Kleber Y. Fertrin, Christine A. Brantner, Mathew P. Daniels, James Nichols, J. Philip McCoy, and Gregory J. Kato. Imaging flow cytometry for automated detection of hypoxia-induced erythrocyte shape change in sickle cell disease. American journal of hematology 89, no. 6 (2014): 598-603.

[2] Taherisadr, Mojtaba, Mona Nasirzonouzi, BehzadBaradaran, AlirezaMehdizade, and I. Shiraz.New approach to red blood cell classification using morphological image processing. Shiraz E-Medical Journal 14, no. 1 (2013): 44-53.

[3] Rakshit, Pranati, and Kriti Bhowmik.Detection of abnormal findings in human RBC in diagnosing G-6PD deficiency HaemolyticAnaemia using image processing. In Condition Assessment Techniques in Electrical Systems (CATCON), 2013 IEEE 1st International Conference on, pp. 297-302. IEEE, 2013

[4] Gonzalez-Hidalgo, Manuel, F. A. Guerrero-Pena, S. Herold-Garcia, AntoniJaume-i-Capó, and P. D. Marrero-Fernandez. Red blood cell cluster separation from digital images for use in sickle cell disease.IEEE journal of biomedical and health informatics 19, no. 4 (2015): 1514-1525

[5] Sahu, Menika, Amit Kumar Biswas, and K. Uma. Detection of Sickle Cell Anemia in Red Blood Cell: A review. International Journal of Engineering and Applied Sciences (IJEAS) 2, no. 3 (2015).

[6] Mahmood, NasrulHumaimi, and Muhammad AsrafMansor. Red blood cells estimation using Hough transform technique. Signal \& Image Processing 3, no. 2 (2012): 53.

[7] Hirimutugoda, Y. M., and GaminiWijayarathna. Image analysis system for detection of red cell disorders using artificial neural networks. Sri Lanka Journal of Bio-Medical Informatics 1, no. 1 (2010).

[8] Sharma V, Rathore A, Vyas G. Detection of sickle cell anaemia and thalassaemia causing abnormalities in thin smear of human blood sample using image processing. In Inventive Computation Technologies (ICICT), International Conference on 2016 Aug 26 (Vol. 3, pp. 1-5).IEEE.

[9] Akrimi, J.A., Suliman, A., George, L.E. and Ahmad, A.R., 2014.November. Classification red blood cells using support vector machine. In Information Technology and Multimedia (ICIMU), 2014 International Conference on (pp. 265-269). IEEE.

[10] Xu, M., Papageorgiou, D.P., Abidi, S.Z., Dao, M., Zhao, H. and Karniadakis, G.E., 2017. A deep convolutional neural network for classification of red blood cells in sickle cell anemia. PLoS computational biology, 13(10), p.e1005746.

[11] V. D. AK, S. Sharmila, Abhishek Kumar, A. K. Bashir, Mamoon Rashid, Sachin Kumar Gupta \&Waleed S. Alnumay. A novel solution for finding postpartum haemorrhage using fuzzy neural techniques.Neural Computing and Applications (2021) (https://doi.org/10.1007/s00521-020-05683-z)

[12] Indhumathi et.al , "Healthcare Management of Major Cardiovascular Disease-A review", 2021 6th International Conference on Inventive Computation Technologies (ICICT), (DOI: 10.1109/ICICT50816.2021.9358519)

[13] S.V. Ruphitha et.al, " Management of Major Postpartum Haemorrhage by using Zigbee protocol - A Review “, 2021 6th International Conference on Inventive Computation Technologies (ICICT) (DOI: 10.1109/ICICT50816.2021.9358757)

[14] V.D.Ambeth Kumar et,al, "Enhancement in Footprint Image using Diverse Filtering Technique" Procedia Engineering journal, Volume 8, No.12, 1072-1080, 2012 . [doi:10.1016/j.proeng.2012.01.965] 